\title{
Coevolution of Synchronization and Cooperation in Costly Networked Interactions
}

\author{
Alberto Antonioni ${ }^{1,2,3, *}$ and Alessio Cardillo ${ }^{4,3, \dagger}$ \\ ${ }^{1}$ Faculty of Business and Economics, University of Lausanne, CH-1015 Lausanne, Switzerland \\ ${ }^{2}$ Grupo Interdisciplinar de Sistemas Complejos (GISC), Departamento de Matemáticas, \\ Universidad Carlos III de Madrid, E-28911 Leganés, Madrid, Spain \\ ${ }^{3}$ Institute for Biocomputation and Physics of Complex Systems (BIFI), University of Zaragoza, E-50018 Zaragoza, Spain \\ ${ }^{4}$ Laboratory for Statistical Biophysics, École Polytechnique Fédérale de Lausanne (EPFL), CH-1015 Lausanne, Switzerland
}

(Received 25 October 2016; revised manuscript received 17 March 2017; published 8 June 2017)

\begin{abstract}
Despite the large number of studies on synchronization, the hypothesis that interactions bear a cost for involved individuals has seldom been considered. The introduction of costly interactions leads, instead, to the formulation of a dichotomous scenario in which an individual may decide to cooperate and pay the cost in order to get synchronized with the rest of the population. Alternatively, the same individual can decide to free ride, without incurring any cost, waiting for others to get synchronized to his or her state. Thus, the emergence of synchronization may be seen as the byproduct of an evolutionary game in which individuals decide their behavior according to the benefit-to-cost ratio they accrued in the past. We study the onset of cooperation and synchronization in networked populations of Kuramoto oscillators and report how topology is essential in order for cooperation to thrive. We also display how different classes of topology foster synchronization differently both at microscopic and macroscopic levels.
\end{abstract}

DOI: 10.1103/PhysRevLett.118.238301

The simultaneous occurrence of events known as synchronization constitutes one of the most fascinating phenomenon ever studied. Synchronization has, in fact, been observed in systems of different nature, from power grids to biological and chemical environments, just to name a few [1-4]. If the system is composed of many units, the pattern of interactions among them plays a cornerstone role in the establishment of the conditions under which the onset of synchronization occurs $[5,6]$. Initially, scientists have studied such onset by encoding interactions as either a mean-field or a regular lattice $[1,4]$. However, interactions in real systems are better described as complex networks [7], and therefore, it is important to pursue the study of synchronization within such a paradigm [5].

Despite the abundance of studies regarding the emergence of synchronization in networked populations of dynamical units, a key aspect has been neglected thus far: the existence of a cost associated with the interactions responsible for the update of the state of each dynamical unit. Yet, it seems reasonable to assume that the introduction of a cost affects the dynamics. With the present Letter, we sought to highlight what happens to the synchronization phenomena when interactions between individuals are regulated by an evolutionary noncooperative game. More specifically, we are interested in studying the emergence of synchronization in systems where agents can decide whether to interact-or not-with their neighbors by evaluating the cost sustained to alter their own state and the benefit received by getting more synchronized with the rest of the neighborhood. Decision processes based on the evaluation of a payoff constitute the heart of evolutionary game theory $[8,9]$. Thus, a coevolutionary approach based on synchronization and evolutionary game theory is the natural frame set for studying this kind of problem. We are interested in assessing under which conditions the will of getting synchronized (i.e., cooperating) affects the attainment of a global synchronized state. In addition, since the topology of interactions plays a role of paramount importance for the onset of a collective behavior [5,10,11], we also want to study the nexus between different topologies and the thriving of cooperation and synchronization.

In the proposed model, we study the emergence of synchronization and cooperation in networked populations of coupled Kuramoto oscillators [6,12-14]. Each node corresponds to a different agent or oscillator called to face a dilemma, named the evolutionary Kuramoto Dilemma, whenever it must decide the strategy to adopt in the future. As we will show, the structure of interactions dramatically affects the conditions under which cooperation and synchronization thrive. Specifically, we observe the onset of a new transition from the synchronized to incoherent state when either the coupling strength or the relative cost becomes too elevated. Moreover, the location of the transition nontrivially depends on the topology of the interaction structure.

Let us consider a graph $G(N,\langle k\rangle)$ of $N$ nodes and average degree $\langle k\rangle$. Each node, $l$, represents a dynamical unit, and its state is characterized by its strategy $s_{l}$, phase $\theta_{l}$, and natural frequency $\omega_{l}$. The strategy is equal to $s_{l}=1$ $\left(s_{l}=0\right)$ if the agent is a cooperator (defector) and shapes the interaction pattern of the agent. The evolution of the phase is governed by a slightly modified version of the Kuramoto model [12-14] given by 


$$
\dot{\theta}_{l}=\omega_{l}+s_{l} \lambda \sum_{j=1}^{N} a_{l j} \sin \left(\theta_{l}-\theta_{j}\right),
$$

where $\lambda$ is the coupling strength, and $a_{l j}$ are the elements of the adjacency matrix $\mathcal{A}$ of $G$. Initially, both $\theta$ and $\omega$ are uniformly distributed over the interval $[-\pi, \pi]$. To gauge the global synchronization level of the system, we use the Kuramoto order parameter $r_{G}[12,13]$, given by

$$
r_{G} e^{i \Psi}=\frac{1}{N} \sum_{j=1}^{N} e^{i \theta_{j}} \quad \text { with } \quad r_{G} \in[0,1],
$$

where $i$ is the imaginary unit and $\Psi$ is the average phase of the system. When $r_{G}=0$, the system is in the incoherent state where all the oscillators have distinct phases. Conversely, $r_{G}=1$ denotes a fully coherent (i.e., synchronized) state where all the oscillators have the same phase. Apart from the global order parameter, it is possible to define a local measure, $r_{l}$, which only accounts for the level of synchronization of a given node with respect to its neighbors. This, in turn, stems from the general case of Eq. (2), which, for a pair of nodes $l$ and $m$, is

$$
r_{l m} e^{i\left(\theta_{l}+\theta_{m}\right) / 2}=\frac{e^{i \theta_{l}}+e^{i \theta_{m}}}{2} .
$$

Hence, for a node $l$, the local order parameter, $r_{l}$, is

$$
r_{l}=\frac{\sum_{m=1}^{N} a_{l m} r_{l m}}{\sum_{m=1}^{N} a_{l m}}
$$

Consequently, we can define its average over all the nodes as $r_{L}=(1 / N) \sum_{l} r_{l}$.

The evolution of the strategy of a given node or player $l$ depends on the evaluation of the payoff accumulated during one discrete step of synchronization dynamics. The payoff is given by the difference between the benefit $b_{l}$ and the $\cos t c_{l}$ that the player attains. The former accounts for how much an oscillator has converged towards being synchronized with its neighbors and is equal to the local order parameter $r_{l}$, given by Eq. (4). The latter is given by the absolute value of the angular acceleration, i.e.,

$$
c_{l}=\Delta \dot{\theta}_{l} \equiv\left|\dot{\theta}_{l}(t)-\dot{\theta}_{l}(t-\epsilon)\right|,
$$

where $\epsilon=0.01$ is the discrete step used to compute the synchronization dynamics given by Eq. (1) with the RungeKutta method. The absolute value is required to ensure that $c_{l}$ is always semidefinite positive. Finally, the payoff of a node is defined as

$$
\Pi_{l}=r_{l}-\alpha \frac{c_{l}}{2 \pi} .
$$

Here, the cost has been divided by $2 \pi$ to make it commensurable with the benefit. Furthermore, we modulate the role of the cost by multiplying it for a scalar $\alpha$ named relative cost. The payoff function is designed in a way such that the benefit complies with the assumption that nodes with larger degrees could accumulate a higher payoff and, also, that low levels of synchronization may lead to negative payoffs. Once the players accumulate their payoffs, they decide the strategy to adopt in the next time step by means of the so-called Fermi rule $[9,11,15]$. In such a rule, the focal player $l$ randomly selects one of its neighbors, $m$, and adopts its strategy with a probability given by

$$
P\left(s_{l} \leftarrow s_{m}\right)=\frac{1}{1+e^{-\beta\left(\Pi_{m}-\Pi_{l}\right)}},
$$

where $\beta$ accounts for the "irrationality" of the players. Without loss of generality, we use $\beta=1$ throughout this Letter. We also point out that our results are qualitatively in agreement with those using other update rules [11], see Supplemental Material (SM), Sec. VI [16]. We consider networks with $N=1000$ and $\langle k\rangle=6$. As an initial condition, we set half of the population made of cooperators. After each synchronization step, agents accumulate their payoffs and synchronously update their strategies according to Eq. (7). We repeat these steps until the system reaches the stationary state.

To summarize, a cooperator always accepts to interact with its neighbors trying to reach mutual synchronization by paying the cost associated with such interactions. A defector, instead, refuses to interact with its neighbors and does not pay any cost. Given this scenario, we can consider a well-mixed population initially composed of $(N-1)$ cooperators plus a single defector. From an evolutionary point of view, the defector is highly advantaged obtaining, on average, the same benefit of all other members (getting synchronized) without incurring any cost. Thus, the defector has a payoff always greater than a cooperator, despite the fact that cooperating would imply a higher benefit for the entire population. Hence, the system evolutionarily undergoes a transition to complete defection, which, in the jargon of evolutionary game theory, is called the tragedy of the commons $[8,17]$.

As we have seen, the well-mixed scenario leads to the emergence of a complete non-cooperative and incoherent state. On the other hand, it has been reckoned that the structure of interactions is one of the mechanisms fostering the emergence of both cooperation and synchronization $[18,19]$. In light of that, we sought whether networked interactions might overcome the temptation to defect and, in turn, lead to the emergence of a coherent state. Therefore, it is interesting to study under which circumstances networked populations allow synchronization to thrive. To explore such a scenario, we choose three different network topologies, namely, Erdős-Rényi (ER) random graphs, random geometric graphs (RGG), and BarabásiAlbert (BA) scale-free networks [7,20-22]. 

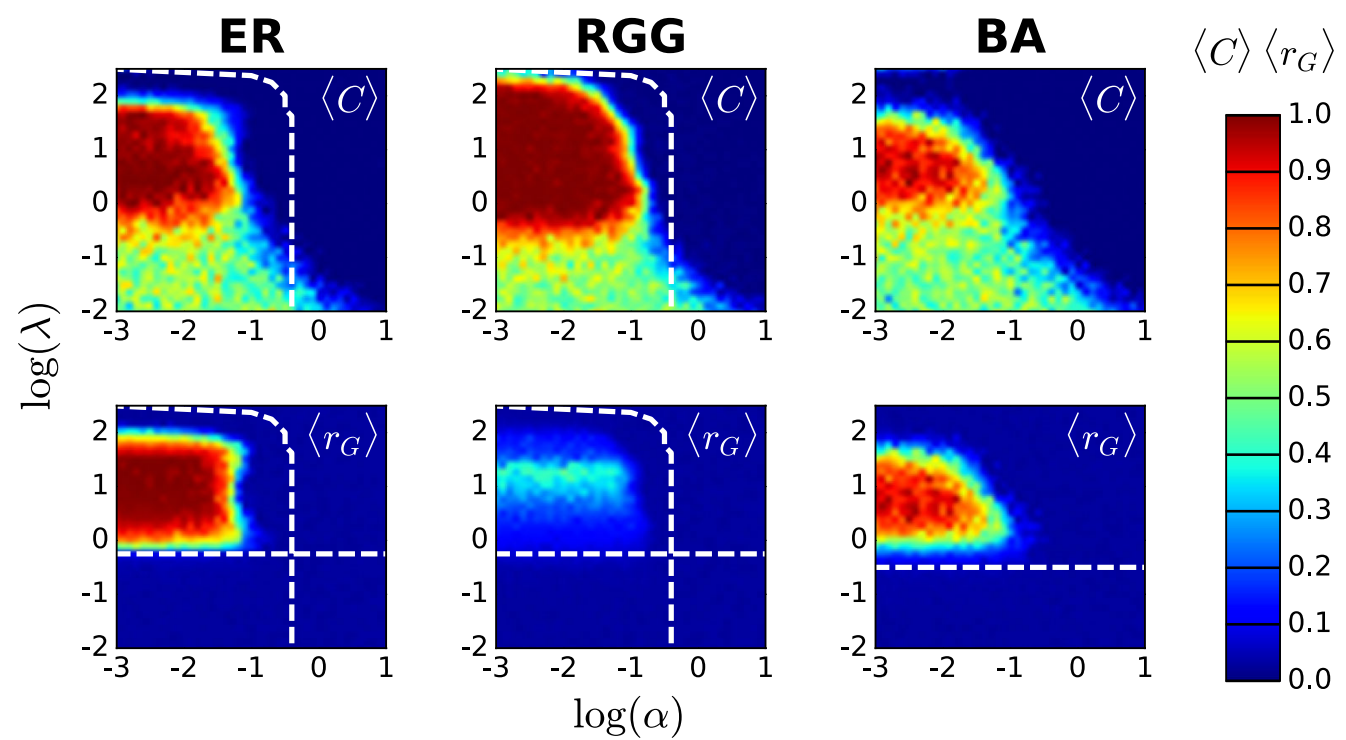

FIG. 1. Emergence of cooperation and synchronization at global scale. The top (bottom) row illustrates the average level of cooperation (synchronization) $\langle C\rangle\left(\left\langle r_{G}\right\rangle\right)$ as a function of the coupling $\lambda$ and relative cost $\alpha$. Each column corresponds to a different topology, namely, ER, RGG, and BA. Results are averages over 50 different realizations.

We begin the study of our toy model by looking at its global behavior. In Fig. 1, we display both the average level of cooperation, $\langle C\rangle$ (top), and the global order parameter, $\left\langle r_{G}\right\rangle$ (bottom), as a function of the relative cost $\alpha$ and coupling strength $\lambda$. At first glance, we notice a strong correlation between the onset of cooperation and synchronization due to the intertwining among these dynamics. For example, for low and intermediate values of $\alpha$, the system displays a transition from an incoherent state to a fully coherent one when the coupling exceeds a critical value. It is worth mentioning that such a transition takes place at couplings slightly above the expected ones given by $\lambda_{c}^{\text {theor }}=\lambda_{c}^{\mathrm{MF}}\left(\langle k\rangle /\left\langle k^{2}\right\rangle\right)$, where $\lambda_{c}^{\mathrm{MF}}$ is the critical value of the coupling in the mean field (MF) case [18]. For each topology, we display $\lambda_{c}^{\text {theor }}$ as a horizontal dashed line. For low relative costs, as the coupling increases, we observe the appearance of a second spontaneous transition from a coherent state to an incoherent one. The reason behind such a loss of synchronization is the increasing burden associated with the variation of the angular speed, i.e., the cost, with respect to the benefit associated to the increase of synchronization. Such a transition is observed exclusively for small to intermediate values of $\alpha$ and the region where synchronization takes place shrinks as $\alpha$ increases. Ultimately, for high values of $\alpha$, synchronization never occurs independently of the coupling strength and of the considered topology. In the case of homogeneous networks, following the method introduced by Ohtsuki et al. [23] (see SM, Sec. II [16]), we can analytically perimeter the region of the parameter space inside which a single defector is able to invade a population of cooperators. Such a region is delimited by the curved dashed line in the ER and RGG panels. In analogy with other studies on coevolutionary dynamics [24], the results shown in Fig. 1 tell us that BA networks do not promote synchronization and cooperation more than ER random graphs; in spite of what was reported previously for each dynamics $[5,18,25,26]$. Yet, RGG panels point towards the existence of additional mechanisms associated with spatial correlations and the presence of community structure in order to converge to more cooperative outcomes (see SM, Sec. IV [16]).

To shed light on such a discrepancy, in Fig. 2, we present a microscopic analysis by measuring $\left\langle r_{L}\right\rangle_{k_{\sigma}}$ for different connectivity classes, $k_{\sigma}$, and for three scenarios of relative cost. More precisely, we consider the following cases: $\alpha=10^{-3}, 10^{-1.4}, 1.0$, to account for cheap, intermediate, and expensive interactions, respectively. Darker solid lines account for low degree nodes while brighter lines account for highly connected ones. The vertical dashed lines delimit the region where synchronization theoretically emerges (dark red) and vanishes (cyan). The horizontal line, instead, accounts for the analytical value that $r_{L}$ assumes when two nodes are randomly selected in a well-mixed population (see SM, Sec. I [16]) accounting for a scenario where no synchronization is observed. A closer inspection to Fig. 2 reveals a set of intriguing features. The first is that in BA networks - and also, to a large extent, in ER ones—nodes tend to attain synchronization all together, in accordance with [18], but then they lose it in a hierarchical order, instead. This is not the case for the RGG where a hierarchy exists in both transitions, whose quantitative analysis deserves further investigation. Moreover, there is a value of $\lambda$ for which the hierarchy gets inverted, meaning that nodes placed at the core of communities tend to act as condensation nuclei exerting a positive feedback for the formation of cooperative clusters, but are also burdened 

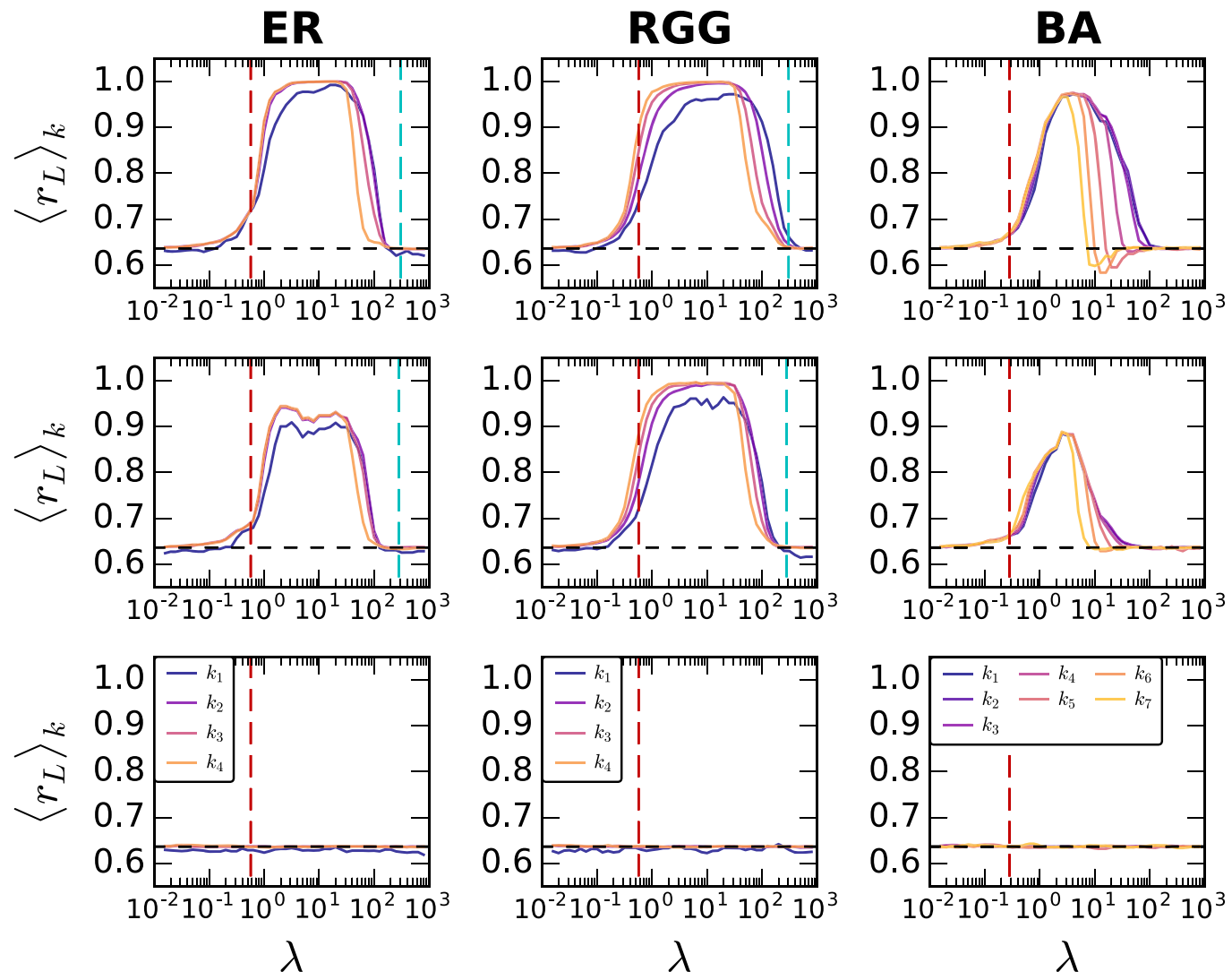

FIG. 2. Emergence of cooperation and synchronization at microscopic scale. Each plot displays the average local Kuramoto parameter for different degree classes $\left\langle r_{L}\right\rangle_{k_{\sigma}}$ as a function of the coupling $\lambda$ and for the three studied topologies (ER, RGG, BA). Colors refer to different degree classes: $k_{\sigma} \in\left\{k_{1}, \ldots, k_{4} \Leftrightarrow[1,3],[4,7],[8,10],[11, \infty]\right\}$ for both ER and $\mathrm{RGG}$, and $k_{\sigma} \in\left\{k_{1}, \ldots, k_{7} \Leftrightarrow\right.$ $[1,5],[6,10],[11,20],[21,40],[41,55],[56,80],[81, \infty]\}$ for BA. Each row corresponds to a different value of $\alpha$ : top row, $\alpha=10^{-3}$, middle row, $\alpha=10^{-1.4}$, and bottom row, $\alpha=1.0$. Results are averages over 50 different realizations.

with higher costs and, therefore, lose synchronization sooner (see SM, Sec. IV [16]). The loss of synchronization is particularly strong in BA networks and is usually accompanied by a drop of $r_{L}$ below the average value in the low-cost interaction regime. The intermediate-cost regime presents a similar picture, but the second transition shrinks. Finally, in the high-cost regime, we also observe the complete absence of synchronization at a local level. The average cooperation level essentially follows the same trend of the local synchronization (see SM, Sec. III [16]). We also performed simulations to test the robustness of our results for different initial conditions, letting the system synchronize for $\tau$ steps before starting the coevolutionary process, and a higher initial fraction of cooperators (see SM, Sec. V [16]).

In conclusion, by accounting for the existence of a cost associated with synchronization interactions, we have built a coevolutionary toy model based on the intertwining of synchronization and evolutionary game theory. The resulting dilemma has been named the evolutionary Kuramoto Dilemma. Network reciprocity has proven itself as a valuable catalyzer of both the survival of cooperation and synchronization, and the effects of accounting for the cost of interaction in networked populations are twofold. On one hand, high values of the coupling result in the desynchronization of the system, which has been previously observed only in higher-order models such as the Rössler model [27]. The other result is the appearance of a hierarchy in the degree of nodes attaining or losing synchronization, which depends on the topology in a nontrivial manner. Our numerical results have been performed for a relatively small system size, although analogous chimera states can also emerge on larger RGG and lattice topologies [28,29]. Considering more realistic patterns, like spatial proximity and heterogeneous ones, sheds some light on such dependence and paves the way to further investigations. Finally, the model presented hereapart from looking at synchronization under a different perspective - can be used to tackle a wide range of problems, leveraging the interplay among coupling, cost, and topology to drive the system towards a coherent or incoherent state. For example, the relation between cooperation and synchronization has been explored in the context of social, cognitive, and behavioral sciences. In the former, previous studies pointed at assessing whether the execution of coordinated or synchronized actions may foster the onset of cooperation (military drills are an example of such situations) [30-32]. In the latter, instead, studies aimed at understanding the individual's 
behavioral plasticity-i.e., the will of individuals to disregard their individual preferences - could be altered to improve the synchronization [33-37]. An even more appealing application is represented by the so-called "social insects" (ants, bees, fireflies, etc.) whose synchronized (or not) behavior-i.e., the flashing of fireflies and the Leptothorax acervorum ants activity cycle to cite a few-can be explained in terms of natural selection [38-42]. Last, a modified version of the Kuramoto model has also been used to study the attainment of consensus in opinion dynamics [43-45].

The authors thank M. Frasca, J. Gómez-Gardeñes, and P. De Los Rios for helpful discussions and valuable comments. The authors acknowledge the financial support of the Swiss National Science Foundation under Grant No. CRSII2_147609 (A. C.), and Grants No. P2LAP1161864 and P300P1-171537 (A. A.).

*alberto.antonioni@unil.ch †alessio.cardillo@epfl.ch

[1] A. Pikovsky, M. Rosenblum, and J. Kurths, Synchronization: A Universal Concept in Nonlinear Sciences (Cambridge University Press, Cambridge, England, 2003).

[2] A. E. Motter, S. A. Myers, M. Anghel, and T. Nishikawa, Nat. Phys. 9, 191 (2013).

[3] F. Varela, J.-P. Lachaux, E. Rodriguez, and J. Martinerie, Nat. Rev. Neurosci. 2, 229 (2001).

[4] R. E. Mirollo and S. H. Strogatz, SIAM J. Appl. Math. 50, 1645 (1990).

[5] A. Arenas, A. Díaz-Guilera, J. Kurths, Y. Moreno, and C. Zhou, Phys. Rep. 469, 93 (2008).

[6] F. A. Rodrigues, T. K. DM. Peron, P. Ji, and J. Kurths, Phys. Rep. 610, 1 (2016).

[7] S. Boccaletti, V. Latora, Y. Moreno, M. Chavez, and D. Hwang, Phys. Rep. 424, 175 (2006).

[8] J. M. Smith, Evolution and the Theory of Games (Cambridge University Press, Cambridge, England, 1982).

[9] L. E. Blume, Games Econ. Behav. 5, 387 (1993).

[10] A. Barrat, M. Barthélemy, and A. Vespignani, Dynamical Processes on Complex Networks (Cambridge University Press, Cambridge, England, 2008).

[11] C. P. Roca, J. A. Cuesta, and A. Sánchez, Phys. Life Rev. 6, 208 (2009).

[12] Y. Kuramoto, Prog. Theor. Phys. Suppl. 79, 223 (1984).

[13] S. H. Strogatz, Physica (Amsterdam) 143D, 1 (2000).

[14] J. A. Acebrón, L. L. Bonilla, C. J. Pérez Vicente, F. Ritort, and R. Spigler, Rev. Mod. Phys. 77, 137 (2005).

[15] G. Szabó and C. Tóke, Phys. Rev. E 58, 69 (1998).

[16] See Supplemental Material at http://link.aps.org/ supplemental/10.1103/PhysRevLett.118.238301 for detailed estimation of the conditions for the onset of synchronization and cooperation, further analysis on the RGG case, and additional results on different initial conditions and update rules.

[17] G. Hardin, Science 162, 1243 (1968).

[18] J. Gómez-Gardeñes, Y. Moreno, and A. Arenas, Phys. Rev. Lett. 98, 034101 (2007).

[19] M. A. Nowak, Science 314, 1560 (2006).

[20] P. Erdôs and A. Rényi, Publicationes Mathematicae (Debrecen) 6, 290 (1959).

[21] J. Dall and M. Christensen, Phys. Rev. E 66, 016121 (2002).

[22] A. L. Barabási and R. Albert, Science 286, 509 (1999).

[23] H. Ohtsuki, C. Hauert, E. Lieberman, and M. A. Nowak, Nature (London) 441, 502 (2006).

[24] G. E. Leventhal, A. L. Hill, M. A. Nowak, and S. Bonhoeffer, Nat. Commun. 6, 6101 (2015).

[25] F. C. Santos and J. M. Pacheco, Phys. Rev. Lett. 95, 098104 (2005).

[26] F. C. Santos, J. M. Pacheco, and T. Lenaerts, Proc. Natl. Acad. Sci. U.S.A. 103, 3490 (2006).

[27] S. Yanchuk, Y. Maistrenko, and E. Mosekilde, Physica D (Amsterdam) 154D, 26 (2001).

[28] D. M. Abrams and S. H. Strogatz, Phys. Rev. Lett. 93, 174102 (2004).

[29] M. J. Pannagio and D. M. Abrams, Nonlinearity 28, R67 (2015).

[30] S. S. Wiltermuth and C. Heath, Psychol. Sci. 20, 1 (2009).

[31] D. Lakens and M. Stel, Soc. Cogn. 29, 1 (2011).

[32] J. Bruggeman, arXiv:1312.6809.

[33] J. E. Herbert-Read, S. Krause, L. J. Morrell, T. M. Schaerf, J. Krause, and A. J. W. Ward, Proc. R. Soc. B 280, 20122564 (2013).

[34] P. Słowiński, C. Zhai, F. Alderisio, R. Salesse, M. Gueugnon, L. Marin, B. G. Bardy, M. di Bernardo, and K. Tsaneva-Atanasova, J. R. Soc. Interface 13, 20151093 (2016).

[35] F. Alderisio, B. G. Bardy, and M. Di Bernardo, Biol. Cybern. 110, 151 (2016).

[36] F. Alderisio et al. arXiv:1607.02175.

[37] K. Yokoyama and Y. Yamamoto, PLoS Comput. Biol. 7, e1002181 (2011).

[38] E. Bonabeau, G. Theraulaz, J.-L. Deneubourg, S. Aron, and S. Camazine, Trends in Ecology \& Evolution 12, 188 (1997).

[39] D. J. T. Sumpter, Phil. Trans. R. Soc. B 361, 5 (2006).

[40] W. D. Hamilton, J. Theor. Biol. 7, 1 (1964).

[41] J. Buck and E. Buck, Sci. Am. 234, 74 (1976).

[42] S. Boi, I. D. Couzin, N. Del Buono, N. R. Franks, and N. F. Britton, Proc. R. Soc. B 266, 371 (1999).

[43] A. Pluchino, V. Latora, and A. Rapisarda, Int. J. Mod. Phys. C 16, 515 (2005).

[44] A. Pluchino, S. Boccaletti, V. Latora, and A. Rapisarda, Physica (Amsterdam) 372A, 316 (2006).

[45] C. Castellano, S. Fortunato, and V. Loreto, Rev. Mod. Phys. 81, 591 (2009). 